\title{
"Ti saluto, vado in Abissinia". Parma e Africa Orientale tra colonialismo e post-colonialismo. Archivio, didattica e ricerca storica
}

\section{Introduzione}

Cooperazione internazionale e ricerca storica, attività umanitaria e memoria. Apparentemente sono settori che sembrano appartenere a mondi diversi, apparentati - nell'immaginario comune - forse solo dalla volontà dei rispettivi operatori di fare cultura. Questo progetto prova appunto a rendere complementari questi mondi, rispondendo anche alle esigenze di una maggiore comprensione dei complessi fenomeni umani come la migrazione, i rapporti con i paesi e le popolazioni ex-colonizzate, la subalternità, l'alterità e così via. Avviato nel corso del 2017, il progetto - sostenuto dal comune di Parma - nasce dalla collaborazione tra l'Istituto storico della Resistenza e dell'età contemporanea di Parma (Isrec) e l'ong Parma per gli altri (Ppa) con lo scopo iniziale di ricostruire, nelle sue diverse forme, i rapporti intercorsi nella storia recente tra la città e le ex colonie italiane del Corno d'Africa (soprattutto Etiopia ed Eritrea) attraverso la creazione di un archivio sulla memoria coloniale e post-coloniale. L'idea ha un importante precedente, si ispira infatti all'esperienza realizzata nel modenese con il contributo - 
anche qui - di organizzazioni umanitarie e di ricerca storica ${ }^{2}$, il cui lavoro è stato in parte raccolto nella pubblicazione Sognando l'impero. Modena-Addis Adeba (1935-1941) [Bertella Farnetti 2007].

All'obiettivo - assai stimolante - di costruzione di un archivio si è affiancato ben presto quello di realizzare percorsi didattici che partissero dal nucleo di storie e materiale raccolto per arrivare ad affrontare in una prospettiva globale i rapporti locali e nazionali con i paesi africani in oggetto.

\section{L’archivio}

Il nostro primo interesse è stato rivolto alle memorie private: attraverso alcuni appelli pubblici, si è chiesto alla cittadinanza di mettere a disposizione fotografie, lettere e racconti che ci aiutassero a tracciare un primo quadro sulla presenza parmigiana in Etiopia ed Eritrea. Grazie soprattutto ai contatti messi a disposizione da Ppa siamo riusciti ad individuare un primo nucleo importante di storie, gravitanti, in un modo o nell'altro, attorno all'esperienza coloniale italiana. Dal

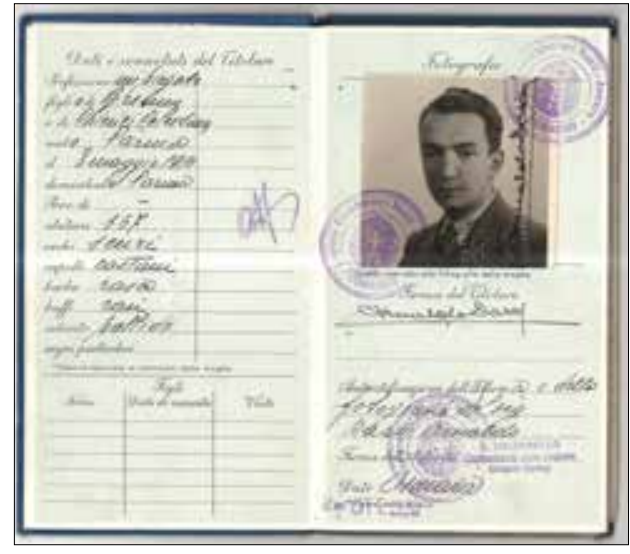

Passaporto di Arnaldo Dazzi (Fondo Arnaldo Dazzi, di proprietà di Giovanna Bottura). punto di vista materiale, siamo venuti in possesso di alcuni fondi fotografici di particolare valore i quali ci offrono squarci sulla vita pubblica, militare e quotidiana coloniale.

Nel caso del fondo Arnaldo Dazzi ${ }^{3}$, ad esempio, abbiamo uno sguardo globale sul vissuto italiano in Africa a cavallo tra gli anni Trenta e Quaranta: i suoi scatti infatti, oltre a immortalare le imponenti parate fasciste, indugiano con velleità antropologiche sulle comunità indigene e sulle loro attività, abbozzandone anche una

Il progetto, nato dall'incontro fra uno storico contemporaneo e due organizzazioni modenesi non profit Modena per gli altri ed Hewa, nel suo sviluppo ha raccolto numerosi aderenti, si rimanda per i dettagli al sito: http://www.memoriecoloniali.org/. 
ritrattistica; a queste due categorie principali si aggiungono numerosi altri aspetti del suo soggiorno africano; il fondo infine si chiude con alcune foto - in parte sfocate - sulle scene di salvataggio dopo l'affondamento della "Nova Scotia".
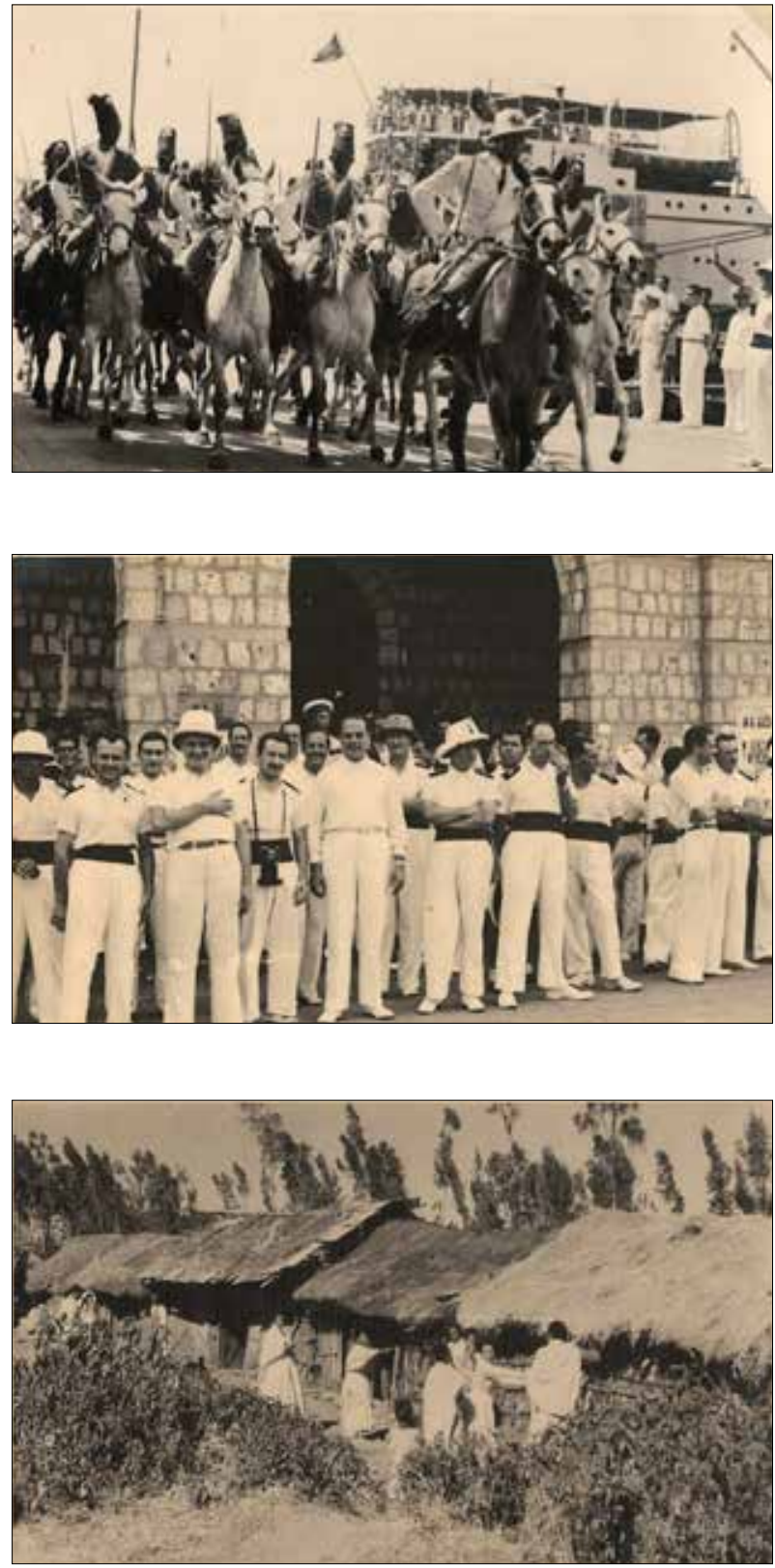

Sul retro della foto vi è l'appunto "Gli "zaptié" a cavallo che precedono la vettura di S.E. Il Gen. Graziani. Massaua, 8.2.1938. XVI» (Fondo Arnaldo Dazzi, di proprietà di Giovanna Bottura)

Arnaldo Dazzi lo si riconosce con la sua inseparabile macchina fotografica. Sul retro della foto vi è l'appunto "In attesa dell'arrivo di S.E. Il Gen. Graziani il giorno del suo imbarco. Massua, 8.2.1938, XVI» (Fondo Arnaldo Dazzi, di proprietà di Giovanna Bottura)

Sul retro della foto vi è l'appunto "Una lite è scoppiata nel vicinato». Nome della località non leggibile, 27.10.1940, XVIII (Fondo Arnaldo Dazzi, di proprietà di Giovanna Bottura) 
Un altro importante gruppo di fotografie ci giunge dal fondo Remo Stocchetti, fotografo di guerra': si tratta di primi piani di dignitari locali ritratti - secondo il racconto del figlio Mario Stocchetti - nell'atto identificativo avvenuto immediatamente dopo aver prestato sottomissione all'Esercito italiano. Che si tratti di un momento di forte tensione emotiva per questi dignitari lo dimostra la straordinaria intensità delle espressioni e delle pose, nelle quali si legge l'eco di un orgoglio piegato.
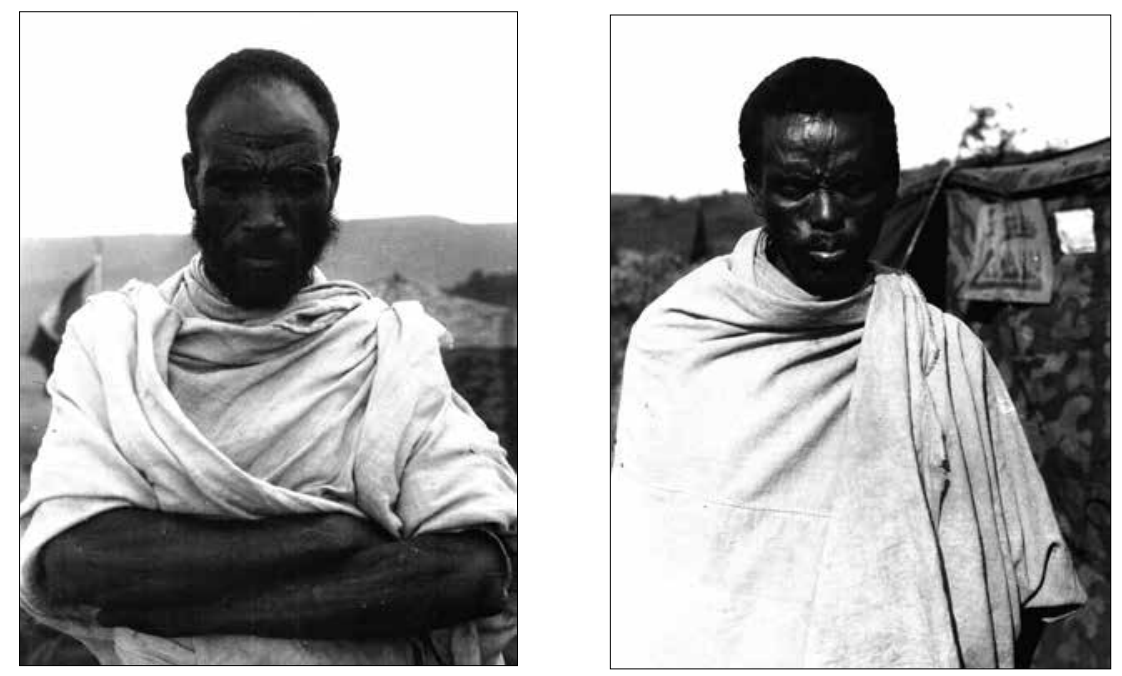

Dignitari locali (Fondo Remo Stocchetti, di proprietà di Mario Stocchetti).

$\mathrm{Ma}$, al di là del tesoro iconografico e materiale a nostra disposizione, sono le storie di vita raccolte che donano profondità all'archivio e più in generale al nostro lavoro di ricerca. I racconti dell'esperienza maturata tra gli anni Trenta e Quaranta risentono il più delle volte degli effetti di una memoria pubblica edulcorata $\mathrm{e}$ spogliata dagli aspetti più nefasti della presenza italiana: ridonda l'immagine di un'Italia portatrice di infrastrutture e di progresso, mentre rimane sullo sfondo la questione razziale, l'uso della violenza e - più in generale - l'intera dimensione colonizzante. Non sembra si tratti, a dire il vero, di una semplice ricezione passiva di una memoria pubblica - per così dire - assolutoria e idealizzante, ma di una sostanziale estraneità, nel quotidiano, ai fatti prevaricanti del colonialismo: in altre parole, l'orizzonte cognitivo di molti civili italiani che giungono 
nel Corno d'Africa dopo la sua (parziale) conquista si esaurisce effettivamente nella maestosa opera edilizia e di sviluppo infrastrutturale che il regime fascista realizza in questi mesi. Un peso rilevante in queste rielaborazioni, infine, deve anche collocarsi sull'eredità culturale e propagandistica - forgiata lungo il Ventennio - che assegnava agli italiani il diritto di prendere quelle terre e di portarvi la propria missione civilizzatrice. Di conseguenza, in molte testimonianze appare raramente esplicitata la consapevolezza di una presenza illeggitima - in questo senso colonizzante - da parte degli italiani. Lo studio della rielaborazione delle memorie individuali, più che i racconti di per sé, risulta essere - in questa prospettiva - uno degli aspetti più interessanti per questo tipo di ricerca. A questo punto però, le storie non bastano da sole. Per dare loro il giusto contesto diviene necessario ricostruire il clima parmense che agì sui sentimenti, le aspettative e i filtri interpretativi dell'esperienza coloniale. Parte integrante della costruzione dell'archivio diviene quindi il risultato della consultazione di giornali, periodici, documenti di prefettura e così via.

Altrettanto stimolante, infine, sono i richiami di queste storie di vita alle dinamiche post-coloniali, per citarne alcuni: il difficile rientro nel parmense dei coloni o, viceversa, $i$ tentativi più meno riusciti di continuare a vivere nelle ormai ex-colonie interfacciandosi con una nuova - e per certi versi ostile - realtà; la condizione d'alterità dei figli di padre italiano e madre africana, il loro rapporto con l'italianità e soprattutto le ricadute del proprio status meticcio sia per chi fosse cresciuto nel paese natìo, sia per chi fosse giunto dopo la guerra in Italia. Nel dopo-
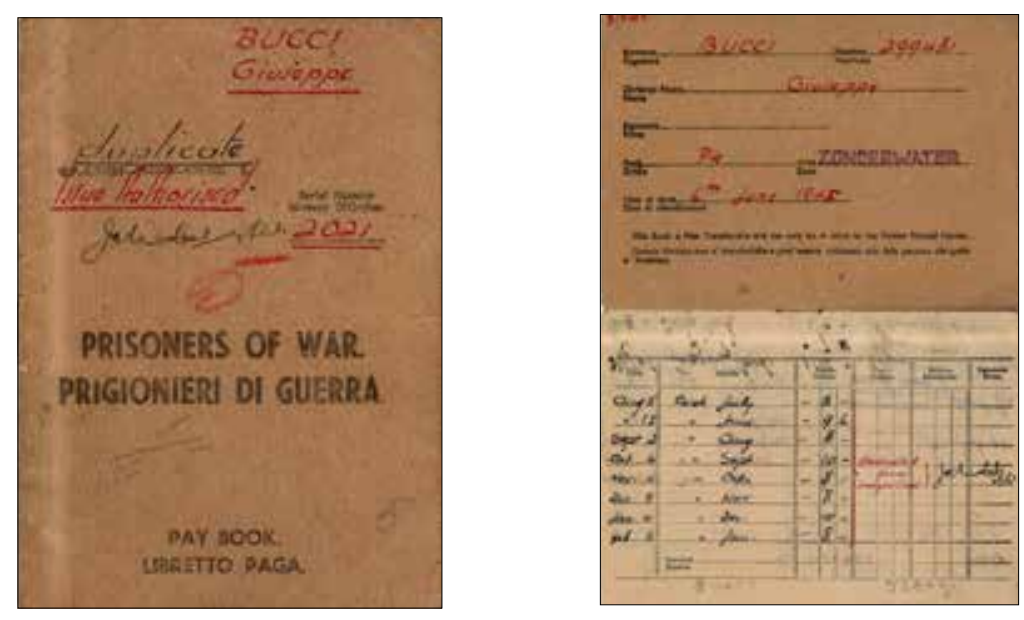

Libretto paga del prigioniero di guerra Giuseppe Bucci, internato nel campo di concentramento inglese di Zonderwater, in Sudafrica (Fondo Giuseppe Bucci, di proprietà di Maurizio Bucci). 
guerra la questione, per questa generazione, si pose sovente in questi termini: troppo bianchi per essere etiopi o eritrei (senza dimenticare il potenziale marchio d'infamia dato dalla propria origine italiana e, quindi, colonizzatrice); troppo scuri per essere italiani, in un'Italia - almeno per quanto riguarda il caso parmense - non ancora abituata alla presenza di persone dal diverso colore della pelle. Proprio il ritorno rappresenta uno dei nodi più intricati di queste storie, un ritorno che in molti casi per i protagonisti o per membri della famiglia fu preceduto da un lungo internamento nei campi di prigionia alleati.

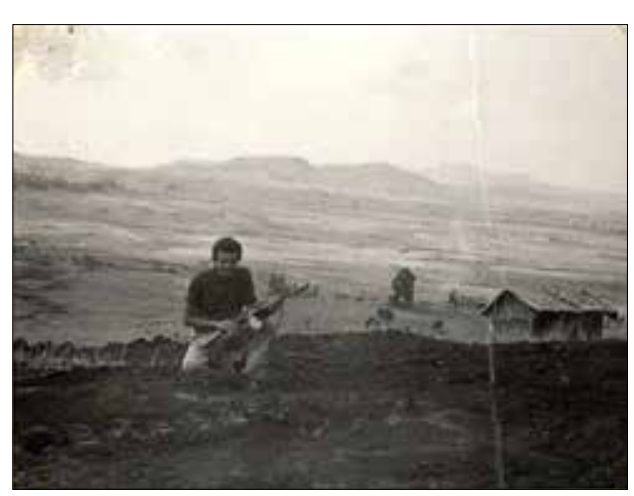

Franco Comacchio, figlio di padre italiano e madre etiope, prende parte alla lotta armata contro il governo militare Derg, in una foto degli anni Settanta (Fondo Franco Comacchio)
Ma le complessità delle testimonianze raccolte - e da raccogliere - sui rapporti tra Parma e i paesi del Corno d'Africa non si esaurisce nell'esperienza coloniale e sulle sue ricadute: dalle avventure imprenditoriali fino alle prime forme di solidarietà organizzata - di cui Ppa è tra le maggiori espressioni - si giunge ai giorni nostri, formando quel mosaico di relazioni, scambi e conflittualità che deve necessariamente essere incluso nel lavoro di costruzione di questo archivio.

\section{La didattica}

Dinanzi a questo primo nucleo di materiale raccolto si è deciso di realizzare percorsi didattici da proporre alle scuole di secondo grado che affrontassero la tematica generale del progetto in una prospettiva ampia. La struttura generale di questi percorsi prevede una parte storiografica ${ }^{5}$ e una più strettamente legata all'ambito della cooperazione internazionale e della convivenza tra popoli diver$\mathrm{si}^{6}$. Per la prima fase, oltre a offrire agli studenti un inquadramento generale sul colonialismo e dei rapporti storici tra Europa e Africa, si è scelto di costruire una narrazione sul materiale d'archivio raccolto impostando i laboratori in un ottica di simulazione di ricerca storica, ovvero, fornendo alla classe le fonti primarie 
che sostengono l'impianto narrativo e stimolando la loro rielaborazione in loco. Particolare attenzione, inoltre, viene rivolta alla rappresentazione propagandistica e soprattutto cinematografica dell'esperienza coloniale e del rapporto con le popolazioni colonizzate, sia lungo il Ventennio che durante il dopoguerra. Segue quindi la seconda fase in cui Ppa, dopo aver introdotto l'articolato mondo in cui operano le Ong, da una parte presenta una panoramica delle proprie attività, dall'altra attraverso testimonianze di esponenti della comunità etiope parmense permette agli studenti di avvicinare ancor di più il proprio sguardo sulle vicende affrontate lungo il percorso.

Nei fatti, questo percorso didattico è in fase di conclusione con tre classi di quinta superiore del Liceo Classico "G.D. Romagnosi” di Parma, mentre sarà ripetuto a breve, sotto forma di progetto di alternanza scuola-lavoro, con una quarta superiore del Liceo delle Scienze Umane "A. Sanvitale" di Parma. Gli studenti, da parte loro, al termine degli incontri laboratoriali dovranno realizzare un evento scenico-narrativo aperto alla cittadinanza inteso come personale rielaborazione dell'intero percorso didattico.

\section{Conclusioni}

L'obiettivo principale resta l'ampliamento dell'archivio; al tempo stesso, si prevede di continuare a intrecciare al lavoro di ricerca percorsi di didattica complessi che offrano agli studenti un accesso agevolato ai grandi temi storiografici e contemporanei attraverso lo studio e la rielaborazione di storie vicine $\mathrm{e}$, per così dire, a misura d'uomo. Da segnalare, inoltre, come la memoria dell'esperienza coloniale - almeno nella sua forma privata - sia risultata rispetto alle aspettative molto radicata e capillare: nel pubblicizzare la ricerca, infatti, diversi parmigiani hanno testimoniato di aver avuto nella propria famiglia uno o più membri coinvolti nell'epopea africana.

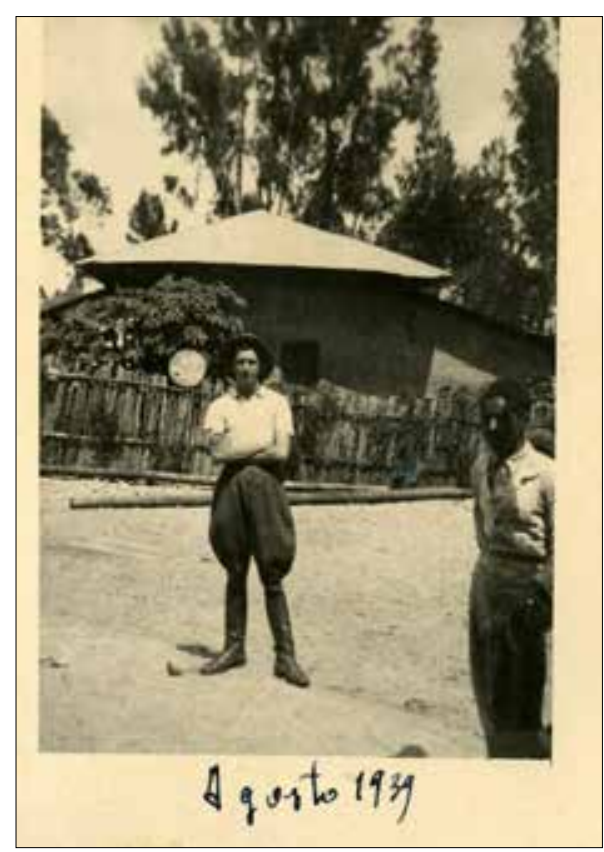

Ritratto di Luigi Bontempi nel 1939 ad Addis Abeba (Fondo famiglia Bontempi, di proprietà di Pier Luigi Bontempi) 
In questo senso si tratta di una memoria forte, anche se sepolta nelle mansarde e nei racconti tramandati di generazione in generazione, in contrasto con una memorialistica pubblica latente. Si può parlare quindi di una memoria da riscoprire. Infine, al di là di eventuali pubblicazioni scientifiche che presentino i futuri risultati di questo lavoro, si prevede come sviluppo naturale della costruzione di questo archivio una mostra che sintetizzi la forza iconografica e narrativa del materiale raccolto, coinvolgendo nella sua realizzazioni - questa è la speranza nuovamente studenti delle scuole di secondo grado di diversi indirizzi.

\section{Bibliografia}

Bertella Farnetti P. 2007, Sognando l'impero: Modena-Addis Adeba (1935-1941), Milano: Mimesis

Isacchini V. 2008, L'onda gridava forte. Il caso del Nova Scotia e di altro fuoco amico su civili italiani, Milano: Mursia

\section{Risorse on line}

Memorie coloniali

http://www.memoriecoloniali.org/

Istituto storico della Resistenza e dell'età contemporanea Parma http://www.istitutostoricoparma.it/

Parma per gli altri Ong http://www.parmaperglialtri.it/it/ 